En nuestro país el $85 \%$ de los adultos mayores dentro de las futuras dos décadas no tendrán derecho a una pensión, así lo ha señalado un estudio realizado por la fundación Saldarriaga Concha y Fedesarrollo, como resultado

\section{La pension familiar: Una norma de papel}

The family pension: A paper standard Aylin Giselle Romero Valeta' - Triana Isabel Deulofeu Castañeda ${ }^{2}$

\author{
$\begin{array}{lll}\text { RECIBIDO: 12-09-16 ACETADO: 24-04-16 } & \text { PUBLICADO: 09-01-17 }\end{array}$
}

Resume

En Colombia, a partir de la ley 1580 de 2012, se instituye la pensión familiar. Esta modalidad pensiona configura una alternativa dingida a salvazuardar el principio de universalidad del sistema pensional ados par obtener la pensión de vejez El presente artículo de investigación pretende abordar la eficacia juríica y la real incidencia que ha tenido esta tipologia de pensión en la población colombiana dentro del sistema de seguridad social integral, yen la busqueda, determinar si la pensión familiar es un intento

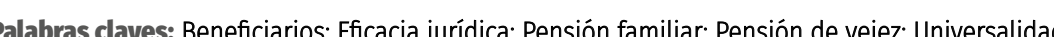

Abstract

Colombia, from the act 1580 of 2012, establishing the family pension. This modality pension sets up an spouses or permanent companions that individually they are unable to obtain the old-age pension. This article of research is intended to address the legal effectiveness and the read $i m$ mact that has had this type

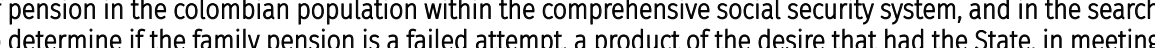
the deficit of the pension system.

Keyword: Beneficiaries; Family pension; Legal effectiveness; Old-age pension; Universality.

de la escasa cobertura pensional producto de un sistema poco incluyente que cada vez dificulta más el acceso a sus beneficios, aumentando las semanas necesarias o la edad requerida para obtener una pensión, pese a que desde el artículo primero de nuestra carta política, Colombia se constituye como un Estado Social de Derecho.

El término "Social" incluido en el artículo primero de la Constitución Política, hace referencia a que: "la acción del Estado debe dirigirse a garantizar a los asociados condiciones de vida dignas y a contrarrestar las desigualdades sociales existentes ofreciendo a todos las oportunidades necesarias para desarrollar sus aptitudes y superar los apremios materiales" de conformidad al criterio de la Corte Constitucional en sentencia SU 747/98.

Con el fin de garantizar dichas condiciones y promover el artículo 48 Constitucional aparece, a partir de la ley 100 de 1993, el Sistema de Seguridad Social Integral, definido como el conjunto de normas, procedimientos e instituciones encaminadas a cubrir las contingencias de carácter económico o en salud que puedan presentar los habitantes del territorio nacional.

Este sistema responde a los principios de eficiencia, solidaridad, integralidad unidad, participación, universalidad, inter alias. Sin embargo, la materialización de esos principios ha sido paulatina, toda vez que en la actualidad, en Colombia más de 20 millones de trabajadores no cotizan para una pensión o que cotizando, no cumplirán los requisitos necesarios para acceder a ella.

La universalidad del Sistema de Seguridad Social Integral se concreta cuando todas las personas de la comunidad participan en sus beneficios. El Estado en aras de materializar los fines propuestos en el artículo segundo de nuestra Constitución Política, debe garantizar la cobertura de las diferentes contingencias que se puedan llegar a presentar durante la vejez a todos los

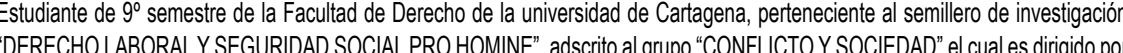
la Dra. Rááalla Ester Sayas Contreras. Correo electrónico. aygiroval121@ @otmail.com

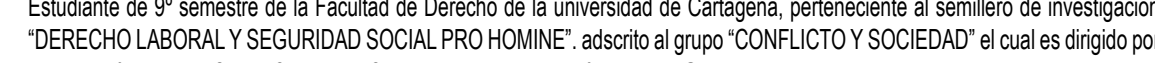
la Dra. Rafaela Ester Sayas Contreras. Correo electrónico. deulofieu.c.trian@@gmail.com

habitantes de un territorio. En la práctica, esto no es completamente viable por razones presupuestales y de infraestructura. 
Por lo tanto, a partir de la de la ley 1580 de 2012 se instituye la pensión familiar, cuya finalidad es que a través de la suma de esfuerzos, miles de parejas colombianas puedan acceder y disfrutar de una pensión sin afectar el equilibrio financiero del sistema.

Es lamentable el estado en el que se encuentran las personas que han culminado el ciclo laboral, que con mucho esfuerzo han realizado los aportes al sistema y que por determinados factores, como el incumplimiento del mínimo legal de semanas a cotizar o un capital acumulado insuficiente, no podrán acceden a la pensión de vejez, quedando en una situación de inseguridad, sujetas a medidas auxiliares no permanentes y sustitutivas de pensión. Es de suma relevancia analizar el impacto que ha tenido la pensión familiar dentro del sistema pensional colombiano.

Frente a esta problemática, la pensión familiar se concibe como una posible alternativa para atenuar la ilusoria idea de acceder al sistema pensional. A pesar que esta institución fue creada hace más de 4 años, el panorama colombiano en materia pensional, dista de haber mejorado.

Por lo anterior, este documento pretende efectuar un análisis en el que se logre contrastar estadísticamente la eficacia de esta modalidad pensional partiendo de la realidad social, y en el camino denotar si la pensión familiar cumple o no el fin por el cual fue instituida.

\section{DÉFICIT DEL SISTEMA PENSIONAL COLOMBIANO}

En Colombia, el sistema pensional padece un déficit coyuntural que se ha extendido por más de tres décadas. Mediante constantes reformas efectuadas al sistema de seguridad social integral, se ha intentado aminorar las consecuencias de un país envejecido y con grandes necesidades en lo que se refiere a la intervención del Estado.

Para 1991 se estimaba un esquema pensional que diera cobertura al 25\% de la población, sin embargo, en el 2007 este porcentaje se mantuvo. En conceptos emitidos por el Ministerio de Trabajo, uno de cada tres colombianos podrá acceder a una pensión, lo que significa que solo el $33 \%$ de la población adulta en edad de retiro o en condición de jubilación logrará pensionarse.
Se estima que la tercera parte de la población mayor de 60 años ha podido pensionarse, y solo el $10 \%$ del total de trabajadores que realizan sus aportes al sistema reunirán las condiciones para ser beneficiarios de la pensión de vejez. (Villar, Forero, Floréz, Puerta, \& Enríquez, 2015)

En el 2016, estudios realizados por Fedesarrollo en alianza con la fundación Saldarriaga Concha, inició un proyecto que tuvo como objetivo principal dar a conocer el proceso de envejecimiento del adulto mayor en Colombia y el reto que debe asumir el Estado al acoger a esta población que aumenta exponencialmente.

La demografía colombiana se distingue por ser abundante y tendiente a la informalidad. El deber que la Constitución Política impuso respecto a la cobertura, desde el principio de progresividad de la seguridad social y el cumplimiento de los fines del Estado, aparentan ser inobservados. En 2017, según cifras del DANE, en el territorio nacional existen 49.167 .120 personas. E Ministerio del Trabajo ha señalado que del total de la población, 22 millones son trabajadores, 7.7 millones cotizan puntualmente al sistema pensional y solo 1,6 millones de estos cotizantes podrán acceder a la pensión de vejez. Lo que evidencia un mal funcionamiento del sistema y una de las tasas más bajas de protección del ciudadano en lo que a seguridad social respecta.

En efecto, el país cuenta con una de las tasas de cobertura más bajas del continente americano, según la oficina de políticas públicas y competitividad corporativa de fomento CAF, cerca de un $32,8 \%$ de cobertura y una desprotección del $57 \%$ respecto a los Servicios de salud y pensiones.

Colpensiones, por un lado, aduce contar con 2.3 millones de afiliados, y solo 230.000 logran cumplir con los requisitos exigidos para ser titular del derecho pensional. El régimen de ahorro individual con solidaridad, por su parte cuenta con 13,2 millones de afiliados y solo 3.3 millones logran obtener una contraprestación fruto de su vida laboral.

La aparente equidad que se prevé en materia pensional es eclipsada por los diagnósticos y resultados del constante estudio del nivel de vida y noción de desarrollo social y económico, imposibilitando el real cumplimiento de las políticas de seguridad social que al tenor de los postulados constitucionales 
de eficiencia, universalidad y solidaridad deberían cubrir progresivamente la totalidad de la población Colombiana.

Para el año 2012, el DANE registro 13.067 millones de personas que no realizaban ninguna actividad laboral, y de estos permanecerán alrededor de 2.269 en la informalidad, número exorbitante que quedará en una incertidumbre jurídica en materia pensional.

Existen multiplicidad de factores que ocasionan el no reconocimiento de la pensión de vejez, como: la dificultad en el acceso; el incumplimiento de los requisitos dispuestos por la ley; el empleo informal; el desempleo; entre otras. Como respuesta a la problemática latente, de forma acelerada, se introduce al ordenamiento jurídico una medida que pretende menguar la inequidad en el acceso a la pensión y potenciar el cobijo de las contingencias de los adultos mayores. Nace a la vida jurídica la pensión familiar como una alternativa para evitar la desprotección y combatir el déficit en la cobertura por parte del Estado en materia pensional. Se torna como una forma de auxilio para la población avejentada que se encuentra lejos de acceder a la pensión de vejez.

Antes de abordar el análisis de la practicidad de la pensión familiar, es necesario describir esta modalidad pensional.

\section{LA PENSIÓN FAMILIAR EN COLOMBIA}

El artículo primero de la ley 1580 de 2012, la define como "aquella que se reconoce por la suma de esfuerzos de cotización o aportes de cada uno de los cónyuges o cada uno de los compañeros permanentes, cuyo resultado es el cumplimiento de los requisitos establecidos para la pensión de vejez en el régimen de prima media con prestación definida o régimen de ahorro individual y de conformidad con lo establecido en la Ley 100 de 1993.

En virtud de lo anterior, vemos como el legislador colombiano intentó ampliar el radio de acción del sistema pensional en cuanto a su cobertura, permitiendo que personas que no cumplan con el lleno de los requisitos exigidos en la ley 100 de 1993 para acceder a la pensión de vejez, puedan conjuntamente, quedar cobijados por el sistema pensional.

Si bien nos hemos referido a la pensión familiar, en el cuerpo del presente documento, como una alternativa para cubrir las contingencias que en la senectud de las personas pudieren presentarse, este aspecto resulta distintivo de la pensión de vejez, lo cierto es que estas dos modalidades constituye diferentes instituciones. La pensión de vejez se obtiene al darse el cumplimiento de unos requisitos específicos, distintos a los exigidos en la pensión familiar, tales como: haber acreditado la relación conyugal o de convivencia permanente por más de cinco años; estar afiliados al mismo régimen; efectuar la sumatoria, entre ambos, del número de semanas exigidas para una pensión de vejez individual o cotizar al sistema de seguridad social en Salud, de conformidad al artículo 204 de la ley 100 de 1993. No obstante, deberán tener en cuenta las especificaciones exigidas para cada régimen pensional.

\section{Pensión familiar en el régimen de prima media con prestación definida.}

Los cónyuges o compañeros permanentes, para acceder a la pensión familiar, deberán cumplir con la edad para pensionarse, que fue modificada por la ley 797 de 2003, bien sea de 57 años para las mujeres y 62 para los hombres. Cabe mencionar que esta modalidad pensional exige, para cada uno de ellos, haber cotizado al menos un $25 \%$ de las semanas a la edad de 45 años.

Como se trata de una modalidad pensional que busca que parejas logren obtener este beneficio a partir de la suma de sus semanas cotizadas, dentro de este régimen, la sumatoria debe equivaler a unas 1300, es decir, 25.5 años. Además, solo aquellas familias que aparezcan dentro en el sistema de identificación y clasificación de potenciales beneficiarios para programas sociales en los niveles 1 y 2, podrán optar por esta prestación, que será de un monto no superior a un salario mínimo legal mensual vigente.

\section{Pensión familiar en el régimen de ahorro individual con solidaridad:}

Dentro de este régimen deberán cumplirse los requisitos establecidos para la devolución de saldos, siendo, la edad mínima y la insuficiencia del capital individual para la pensión de vejez, la sumatoria de esta insuficiencia configuraría el capital necesario para financiar la pensión de vejez; 0 al menos 1.150 producto de la sumatoria de semanas cotizadas. En este régimen la prestación puede exceder de un salario mínimo legal mensual vigente.

Como habíamos mencionado con antelación, al momento de la solicitud de la pensión familiar, los cónyuges o compañeros permanentes deberán estar afiliados al mismo régimen pensional y a la misma administradora. De encon- 
trarse efectuando los aportes a distintos regímenes o fondos de pensiones, uno de ellos deberá solicitar su traslado a la administradora de su cónyuge o compañero permanente con la finalidad de acceder al reconocimiento de la pensión familiar.

Por otra parte, a diferencia de la pensión de vejez, la pensión familiar no constituirá en ningún lugar una prestación individual para cada cónyuge o compañero permanente, por lo que se entiende que se configura una sola pensión a dos beneficiarios. Debemos precisar que el a el artículo 151C dispone que "será una sola pensión, de acuerdo con el artículo 48 de la Constitución Nacional". Asimismo, el contenido descriptivo de esta ley aparenta ser integro. El legislador determina un trámite particular en caso de fallecer uno de los cónyuges o compañeros permanentes; la extinción de esta prestación si fallecidos los cónyuges o compañeros, no existen hijos beneficiarios; igualmente regula en caso de presentarse la separación o el divorcio. En esta medida la Corte Constitucional, en sentencia C-134/16, afirma que "la incompatibilidad de la pensión familiar con otras prestaciones, hace beneficiarios en el régimen de prima media a los clasificados en los niveles 1 y 2 del SISBEN y reafirma que su valor "no podrá exceder de un salario mínimo legal mensual vigente".

Habiendo precisado en la conceptualización de la pensión familiar, nos referiremos en los acápites siguientes en la verdadera incidencia que ha tenido la pensión familiar en la realidad colombiana.

\section{LA VERDADERA CARA DE LA PENSIÓN FAMILIAR}

El Dr. Oscar Iván Palacio Tamayo, investigador en el área de seguridad social, especialmente en el tema de pensión, ha catalogado esta modalidad pensional como un fiasco y una burla. Esta afirmación se justifica en el hecho de que la pensión familiar solo beneficia a un número muy reducido de personas, pues de aproximadamente 400.000 personas que podrían beneficiarse, actualmente solo se han aprobado 175 pensiones.

Colpensiones manifiesta que entre el 30 de diciembre de 2014 y el 30 de abril de 2016, han sido presentadas alrededor de 733 solicitudes de pensión familiar en Colombia.

Nos corresponde entrar a cuestionarnos si la norma que regula la pensión familiar es ineficaz, y a su vez determinar si esa ineficacia se predica por el bajo número de solicitudes efectuadas por los que optan por esta modalidad pensional, o si es consecuencia de la dificultad en el acceso al reconocimiento de las prestaciones contenidas.

Antes de entrar en materia de eficacia jurídica, debemos mirar como luce el panorama en la realidad colombiana.

En el régimen de prima media con prestación definida, como habíamos denotado, el requisito de pertenecer a los niveles 1 y 2 del SISBEN, hace más gravoso el acceso a la pensión familiar.

Solo en Bogotá se presenta la siguiente estadística respecto al SISBEN:

Tabla 1. Estado de aseguramiento población de Bogotá.

\begin{tabular}{|c|c|c|c|}
\hline AFILIADOS POR RÉGIMEN & CANTIDAD & \% DEL TOTAL & \% DE AFLLADOS \\
\hline Activos en Régimen Contributivo & 6.047 .358 & $74,8 \%$ & $80,5 \%$ \\
\hline Suspendidos en Régimen Contributivo & 95.711 & $1,2 \%$ & $1,3 \%$ \\
\hline Afliados Contributivo & 6.143 .069 & $76,0 \%$ & $81,8 \%$ \\
\hline Activos en Régimen Subsidiado & 1.155 .849 & $14,3 \%$ & $15,4 \%$ \\
\hline Regímenes de Excepción (estimado) & 210.294 & $2,6 \%$ & $2,8 \%$ \\
\hline Subtotal afliados & 7.509 .212 & $92,9 \%$ & $100,0 \%$ \\
\hline \multicolumn{2}{|l|}{ SISBEN NO AFILIADOS } & CANTIDAD & \% DEL TOTAL \\
\hline \multicolumn{2}{|c|}{$\begin{array}{l}\text { Afliados al Régimen Subsidiado } \\
\text { (SISBEN Niveles } 1 \text { y } 2+\text { Menores Nivel } 3 \text { ) }\end{array}$} & 29.088 & $0,4 \%$ \\
\hline \multicolumn{2}{|c|}{$\begin{array}{l}\text { Encuestados que no se pueden afliar al subsidiado por puntaje } \\
\text { Sisbén superior }\end{array}$} & 8.305 & $0,1 \%$ \\
\hline \multicolumn{2}{|l|}{ Total SISBEN no afiliado } & 37.393 & $0,5 \%$ \\
\hline
\end{tabular}

Fuente: Recuperada de http//:https://docs.google.com/spreadsheets/d/1Vm5

De acuerdo con la información disponible ofrecida por el Departamento Nacional de Planeación, en septiembre del 2015, el SISBEN tenía 34.964.317 registros validados. Para mayo del 2016 la cifra subió a 35.616 .653 personas, evidenciando que cerca del $75 \%$ de la población nacional pertenece a estos niveles.

Conforme estudios realizados por el DANE ${ }^{1}$ en 2015 , el 94,6\% de las personas manifestaron estar afiliada al Sistema General de Seguridad Social en Salud. 1 Este estudio fue realizado por e I DANE en el año 2015, a través de una Encuesta Nacional de Calidad de Vida -ECV-que tomo como muestra la población colombiana para determinara el número de personas afiliadas al Sistema de Seguridad Social en Salud. 
Para el 2016, el 95,4\% de toda la población nacional manifestó estar afiliada al mismo sistema, arrojando que anualmente incrementa la afiliaciones un 1,5\%.

En virtud de lo anteriormente expuesto, consideramos pertinente determinar cifras que nos permitan dilucidar la magnitud poblacional que podría beneficiarse de la modalidad pensional denominada pensión familiar.

En cifras, sobre la base de la población actual y los porcentajes que el departamento administrativo nacional de estadística coteja anualmente haciendo uso de fórmulas matemáticas ajustadas a cifras demográficas, determinamos que:

Ecuación

$\frac{\text { "total poblacional" }}{\text { "x" }}=\frac{100 \%}{\text { "parte en \%" }} \Rightarrow \quad$ "x" $=\frac{\text { "total poblacional" " "parte en \%" }}{100 \%}$

Tabla 2. Estadística poblacional destinataria de la ley 1580 de 2012

\begin{tabular}{|c|c|c|}
\hline \multicolumn{3}{|c|}{ Estadistica poblacional destinataria de la ley 1580 de 2012} \\
\hline \multicolumn{3}{|c|}{ Poblacion actual Colombiana: $49,167,120$ personas } \\
\hline \multirow{2}{*}{\multicolumn{2}{|c|}{$\begin{array}{l}\text { Mujeres } \\
\text { Hombres } \\
\end{array}$}} & \\
\hline & & \\
\hline \multirow{2}{*}{ Resultados: } & & $998,092,536$ \\
\hline & & \\
\hline
\end{tabular}

consecuencia Colombia crece demográficamente a pasos agigantados, y siendo la población adulta y adulta mayor la que predomina, con un crecimiento anual del $4 \%$ y que para el 2050 la población será de 72 millones, con una expectativa de vida superior a los 79 años y con más del $20 \%$ de los pobladores por encima de 60 años, de acuerdo a cifras arrojadas por el Ministerio de Salud en el año 2010. Es apremiante desenmascarar la pensión familiar como medida contingente del apremio de derechos inagotados.

\section{RECONOCIMIENTO DE LA PENSIÓN FAMILIAR}

El reconocimiento de la pensión familiar debe ser, sin duda alguna, incluyente, eficaz y eficiente. Como logramos ver, estos principios y objetivos no sobresalen en el panorama pensional actual.
Existen 400.000 personas que podrían ser beneficiarias de la pensión familiar, y conforme a la siguiente gráfica, 3 años después de la entrada en vigencia la ley 1580 de 2012, es aprobada la primera solicitud de esta modalidad de pensión en Colombia con una duración de un año y medio de trámite. Colpensiones expresa que anualmente se presentan 735 solicitudes de pensión familiar, es decir, 61.25 solicitudes por mes ante los administradores de fondos pensionales, ya sean de carácter público o privado, de las cuales, de acuerdo al índice determinado en el cuatrimestre 2017, solo son aprobadas 43.75 pensiones familiares. Cerca de 17.5 solicitudes no son aprobadas al mes.

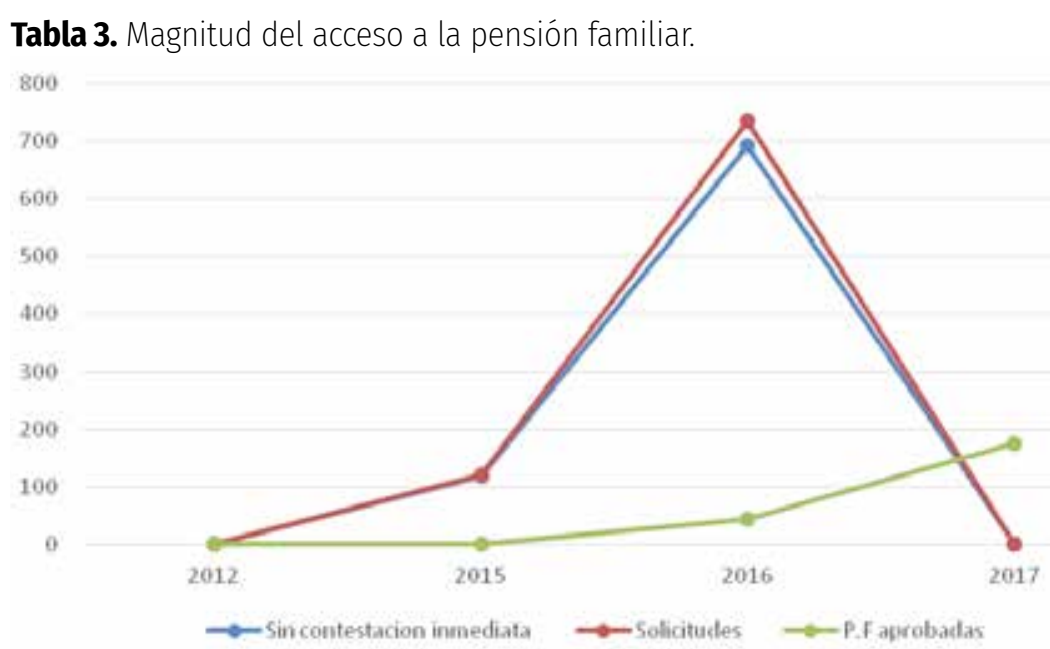

Por: Aylin Romero Valeta y Triana Deultefu Castañeda

\section{EFICACIA JURÍDICA VS LA PENSIÓN FAMILIAR}

Jürgen Habermas, en el análisis multifacético e integrador del Derecho, afirma que no basta que una norma jurídica exista formalmente y pueda coercitivamente ser exigida. Debe darse el cumplimiento de las funciones para las cuales fue creada, y las garantías ofrecidas para la real ejecución de las prescripciones de los derechos contenidos, es decir, sean eficaces.

La realización del Derecho se traduce en la efectividad de la producción de la norma en la sociedad, apreciando la conexión existente entre lo jurídicamente establecido y el hecho social. En otras palabras, la eficacia jurídica hace referencia que la norma jurídica tenga una realización socia.

La pensión familiar, tuvo como finalidad, lograr amortiguar las distintas situaciones que pudieran presentarse durante la etapa de vejez, cuando, personas que pretendan obtener la pensión de vejez, no puedan por multiplicidad de 
factores. El Estado colombiano vio en esta modalidad un respaldo para entrar a rescatar el sistema pensional de una reforma que se pide a gritos.

Como vimos, las cifras son alarmantes. Indiscutiblemente la pensión familiar no está siendo útil en cuanto a la protección de los aspirantes a pensión y mucho menos a contribuir a la reducción del cáncer que ha desarrollado el sistema pensional colombiano.

La pensión familiar debió, mínimamente hasta la fecha, mostrar un gran avance respecto a la cobertura en cuanto al reconocimiento de la prestación, que logra a su vez, incluir en el porcentaje de beneficiarios a la mayoría de las parejas colombiana que cumplan con los requisitos legales, permitiendo que miles de familias logren suplir parte de las necesidades de sus hogares.

Es triste mirar el tan alto porcentaje de personas a la deriva, en la espera de encontrar en la pensión familiar una respuesta para los días por venir y encontrando en el camino procedimientos engorrosos, donde las trabas y la dilación es lo único conocido con certeza.

\section{CONCLUSIÓN}

La pensión familiar, de forma conclusiva, se muestra como una modalidad que logró posicionarse como una alternativa apresurada y mal estructurada por el Estado, esto como consecuencia del porcentaje indicador del reconocimiento de este tipo pensional, siendo significativamente minúsculo, frente al total de potenciales beneficiarios que efectuaron sus aportes al sistema y que desafortunadamente el acceso a la pensión de vejez se torna inalcanzable por múltiples factores

Mauricio Garcia Villegas, como señala en su escrito Normas de Papel, en Colombia existe una cultura de incumplimiento que proviene de parte de los llamados "incumplidores". La realidad social colombiana nos lleva a catalogar la pensión familiar como una norma de papel, pero que en su caso, el incumplimiento surge por parte del Estado, aparentando desconocer el espiritu de la ley 1580 de 2012. Por tal razón, en Colombia urge una modificación en el acceso a la pensión familiar que el Estado, en el ejercicio real y efectivo del poder en aras de la materialización de los fines propuestos en el artículo segundo Constitucional, tiene el deber de recurrir a idear estrategias que logren la verdadera implementación de la pensión familiar, bien sea ampliando el radio de acción o eliminando los procedimientos y requisitos que hacen más gravoso el acceso a la misma.

\section{REFERENCIAS BIBLIOGRAFIA}

Amat, Y. (10 de febrero de 2013). El gobierno destapa plan de reforma de las pensiones. El tiempo.

Amat, Y. (31 de febrero de 2016). Los fondos de pensiones poden reforma al regimen. El tiempo.

Corte constitucional. (16 de marzo de 2016) Sentencia C-134. \{MP. Gabriel Eduardo Mendoza Martelo\}

Corte constitucional. (2 de diciembre de 1998) Sentencia SU-747. (MP. Eduardo Cifuentes) Congreso de Colombia. (23 de diciembre de 1993) ley general del sistema de seguridad social integral \{Ley 100 de 1993\} DO: 41.148

Congreso de Colombia. (1 de octubre de 2012) Ley de pensión familiar \{Ley 1580 de 2012\} DO: 48.570

Congreso de Colombia. (12 de febrero de 2014) decreto reglamentario de la ley de pensión familiar \{decreto 288 de 2014\} DO: 49.062

Constitución política de Colombia \{Const.\} (1991) articulo 48\{Capitulo Il\} vigésima octava edición, Legis

DANE. (2017). poblacion proyectada actual

Floréz, C. E., Villar, L., \& Puerta, N. y. (2015). El proceso de envejecimiento de la poblacion .

Garcia, C. A. (31 de octubre de 2016). Los Fondos de pensiones poden reforma a regimen. El tiempo.

Ministerio de salud. (2010). Demografia y condiciones de vida.

Municipios depuraron la informacion de 240 millones de ciudadanos afiliados sisben. (11 de julio de 2016). Portafolio.

Palacio, o. I. (06 de Abril de 2017). La pensión familiar es un fiasco y una burla". Ambito Juridico.

Viana, J. (15 de Junio de 2016). Sabe qué es una pensión familiar y si puede acceder a ella? Portafolio.

Villar, L., Forero, D., Floréz, C. E., Puerta, N., \& Enríquez, E. (2015). Colombia como envejece Fundacion Saldarriaga y concha. 